\title{
Momentum distributions in medium and heavy exotic nuclei
}

\author{
M. K. Gaidarov, ${ }^{1}$ G. Z. Krumova, ${ }^{2}$ P. Sarriguren, ${ }^{3}$ A. N. Antonov, ${ }^{1}$ M. V. Ivanov, ${ }^{1}$ and E. Moya de Guerra ${ }^{4}$ \\ ${ }^{1}$ Institute for Nuclear Research and Nuclear Energy, Bulgarian Academy of Sciences, Sofia 1784, Bulgaria \\ ${ }^{2}$ University of Ruse, Ruse 7017, Bulgaria \\ ${ }^{3}$ Instituto de Estructura de la Materia, CSIC, Serrano 123, E-28006 Madrid, Spain \\ ${ }^{4}$ Departamento de Fisica Atómica, Molecular y Nuclear, Facultad de Ciencias Fisicas, Universidad Complutense de Madrid, \\ E-28040 Madrid, Spain \\ (Received 15 September 2009; published 11 November 2009)
}

\begin{abstract}
We study nucleon momentum distributions of even-even isotopes of $\mathrm{Ni}, \mathrm{Kr}$, and $\mathrm{Sn}$ in the framework of deformed self-consistent mean-field Skyrme HF + BCS method, as well as of theoretical correlation methods based on light-front dynamics and local density approximation. The isotopic sensitivities of the calculated neutron and proton momentum distributions are investigated together with the effects of pairing and nucleon-nucleon correlations. The role of deformation on the momentum distributions in even-even $\mathrm{Kr}$ isotopes is discussed. For comparison, the results for the momentum distribution in nuclear matter are also presented.
\end{abstract}

DOI: 10.1103/PhysRevC.80.054305

PACS number(s): 21.10.Gv, 21.60.Jz, 21.65.-f, 27.50.+e

\section{INTRODUCTION}

The study of nuclei close to the nuclear drip line and even beyond it has been greatly extended in recent years. This increased interest is based on new phenomena that already have been observed or that are predicted to occur in these nuclei. Since the first experiments [1-6], it has been found from analyses of total interaction cross sections that weakly bound neutron-rich nuclei, e.g., ${ }^{6,8} \mathrm{He},{ }^{11} \mathrm{Li},{ }^{14} \mathrm{Be},{ }^{17,19} \mathrm{~B}$, have increased sizes that deviate substantially from the $R \simeq A^{1 / 3}$ rule. It was realized (e.g., Refs. [7-9]) that such a new phenomenon is due to the weak binding of the last few nucleons that form a diffuse nuclear cloud (the "nuclear halo") due to quantum-mechanical penetration. Another effect is that the nucleons can form a "neutron skin" [3] when the neutrons are on average less bound than the protons. The origin of the skin lies in the large difference of the Fermi energy levels of protons and neutrons so that the neutron wave function extends beyond the effectively more bound proton wave functions [9].

The experiments on scattering of radioactive nuclear beams on proton target at various incident energies (e.g., less than $100 \mathrm{MeV} /$ nucleon for $\mathrm{He}$ isotopes [10-19] and $700 \mathrm{MeV} /$ nucleon for $\mathrm{He}$ and $\mathrm{Li}$ isotopes [20-24]) have allowed one to study the charge and matter distributions of these nuclei using different phenomenological and theoretical methods (e.g., Refs. [18-38]).

As known, the most accurate determination of the charge distributions in nuclei can be obtained from electron-nucleus scattering. For the case of the unstable exotic nuclei the corresponding charge distributions are planned to be studied by colliding electrons with these nuclei in storage rings (see, e.g., the GSI physics program [39] and the plan of RIKEN $[40,41])$. A number of interesting issues can be analyzed by the electron experiments. One of them is to study how the charge distribution evolves with increasing neutron number at fixed proton number or to what extent the neutron halo or skin may trigger sizable changes of the charge root-mean-squared (rms) radius and the diffuseness in the peripheral region of the charge distribution. In Ref. [42] we studied charge form factors of light exotic nuclei $\left({ }^{6,8} \mathrm{He},{ }^{11} \mathrm{Li},{ }^{14} \mathrm{Be},{ }^{17,19} \mathrm{~B}\right)$ using various theoretical predictions of their charge densities. Among the latter we used those from Tanihata et al. [6] for the He isotopes, those from the cluster-orbital shell-model approximation (COSMA) for the $\mathrm{He}$ [28] and $\mathrm{Li}$ [18] isotopes, those from the microscopic large-scale shell-model (LSSM) method (for He [43] and Li [44]), and those from Suzuki et al. [45] for ${ }^{14} \mathrm{Be}$ and ${ }^{17,19} \mathrm{~B}$ nuclei. In Ref. [46] our calculations of the charge form factors of exotic nuclei were extended from light $(\mathrm{He}, \mathrm{Li})$ to medium and heavy nuclei $(\mathrm{Ni}, \mathrm{Kr}$, and $\mathrm{Sn}$ ). For the $\mathrm{He}$ and $\mathrm{Li}$ isotopes the proton and neutron densities obtained in the LSSM method have been used, while for $\mathrm{Ni}, \mathrm{Kr}$, and $\mathrm{Sn}$ isotopes the densities have been obtained in the deformed self-consistent mean-field Skyrme-Hartree-Fock (HF) + BCS method [47-49]. In contrast to the work in Ref. [42], in Ref. [46] we calculated the charge form factors not only within the PWBA but also in DWBA by the numerical solution of the Dirac equation [50-52] for electron scattering in the Coulomb potential of the charge density of a given nucleus. The role of the neutrons has been taken into account. A detailed study of the charge radii and neutron skin in $\mathrm{Ni}, \mathrm{Kr}$, and Sn nuclei, as well as of the formation of the proton skin, has been performed within the same method in Ref. [53].

Another important characteristic of the nuclear ground state is the nucleon momentum distribution (NMD) $n(k)$. The scaling analyses of inclusive electron scattering from a large variety of nuclei (see, e.g., Refs. [54,55] for the $y$ scaling and [56-63] for $\psi^{\prime}$ scaling and superscaling) showed the evidence for the existence of high-momentum components of NMD at momenta $k>2 \mathrm{fm}^{-1}$. It has been shown [60-63] that it is due to the presence of nucleon-nucleon $(N N)$ correlations in nuclei (for a review, see, e.g., Ref. [64]). It has been pointed out that this specific feature of $n(k) / A$ is similar for all nuclei and that it is a physical reason for the scaling and superscaling phenomena in nuclei. As known [64,65], the mean-field approximation (MFA) is unable to describe simultaneously the two important characteristics of the nuclear ground state: the density and momentum distribution. Therefore, a consistent 
analysis of the effects of the $N N$ correlations on both quantities is required using theoretical methods beyond the MFA in the description of relevant phenomena, e.g., the scaling ones. Particular attention has been paid to the NMD in a given single-particle state analyzing the $\left(e, e^{\prime} p\right)$ reactions in nuclei (see, e.g., the review in Ref. [66] and the work in Refs. [67-71] and references therein). The self-consistent density-dependent HF (DDHF) approximation has been applied in Ref. [48] to calculate NMD in spherical and deformed $\mathrm{Nd}$ isotopes, studying the effects of deformation, as well as those of pairing and of dynamical short-range $N N$ correlations.

It is of importance to study the NMD not only in stable but also in exotic nuclei. It is known (see, e.g., Ref. [72]) that in the reactions with exotic nuclei the momentum distribution of a core fragment of the projectile reflects the momentum distribution of the valence nucleons in the projectile near the surface [73]. Many experimental (e.g., Ref. [74]) and theoretical [75] works have been carried out to study the momentum distribution from the breakup of the projectile. For instance, in Ref. [76] the momentum distribution of relative motion between two nucleons has been calculated for both ${ }^{6} \mathrm{He}$ and ${ }^{6} \mathrm{Li}$ two-neutron halo nuclei. The obtained results in the case of realistic $N N$ interaction show two interesting predictions: (i) $S$-wave dominance in the NMD of ${ }^{6} \mathrm{He}$ and (ii) the ${ }^{6} \mathrm{Li}$ momentum distribution is very similar to that of the deuteron. In Ref. [77] the neutron and proton momentum distributions in some stable nuclei $\left({ }^{12} \mathrm{C},{ }^{16} \mathrm{O},{ }^{40} \mathrm{Ca},{ }^{56} \mathrm{Fe}\right.$, and ${ }^{208} \mathrm{~Pb}$ ) were calculated along with those of light neutronrich isotopes of $\mathrm{Li}, \mathrm{Be}, \mathrm{B}$, and $\mathrm{C}$ using the natural-orbital representation (NOR) on the basis of the empirical data for $n(k)$ in ${ }^{4} \mathrm{He}$ and, especially, those for the high-momentum components of the latter.

The main aim of our work here is to calculate the NMD for the same isotopic chains of neutron-rich nuclei $(\mathrm{Ni}, \mathrm{Kr}$, and $\mathrm{Sn})$ for which we had studied charge densities, radii, form factors, halo, and skin in our previous works, Refs. [46] and [53]. The mean-field contributions to $n(k)$ in these nuclei are calculated within the same self-consistent approach applied there in which the one-body energy density functional is obtained starting from a two-body density-dependent Skyrme interaction and a pairing interaction that is treated in the BCS limit. The HF equations are solved for the $(N, Z)$ nucleus using a deformed harmonic-oscillator basis in cylindrical coordinates with oscillator lengths used as variational parameters. The BCS equations are solved at each HF iteration and the occupation numbers are used to construct the density-dependent mean field for the next HF iteration. We refer to this mean-field approach as DDHF + BCS. The remaining effects of the $N N$ interactions, to which we refer as $N N$ correlations, are considered in two of the correlations approaches, namely in the approach (see Refs. $[61,63,78]$ ) using the light-front dynamics (LFD) method (e.g., Ref. [79]) and in that [80] based on the local density approximation (LDA). Several questions are investigated, such as the sensitivity of $n(k)$ to all details of the calculations, e.g., (i) to different types of Skyrme forces, (ii) to the pairing correlation effects, (iii) to the effects of nuclear deformation, and (iv) to the strength of the $N N$ correlations included in the LFD and LDA approaches (respectively, to the values of the correlations strength parameters $\beta$ and $\gamma$ ).
Special attention is paid to the isotopic and isotonic sensitivity of the proton and neutron momentum distributions. The results for $n(k)$ in the exotic nuclei are compared with that in nuclear matter (NM).

The article is organized in the following way. Section II contains the formalism of the deformed Skyrme HF + BCS method and the approaches based on the LFD and LDA methods that provide the model nucleon momentum distributions. The numerical results and discussions are presented in Sec. III. Finally, we draw the main conclusions of this study in Sec. IV.

\section{THEORETICAL FRAMEWORK}

\section{A. Deformed Skyrme HF + BCS formalism}

Some of the results discussed in the next section have been obtained from self-consistent deformed Hartree-Fock calculations with density-dependent Skyrme interaction [49] and pairing correlations. Pairing between like nucleons has been included by solving the BCS equations at each iteration either with a fixed pairing-gap parameter (determined from the odd-even experimental mass differences) or with a fixed pairing-strength parameter. We consider in this article the Skyrme force SLy4 [81] that gives an appropriate description of bulk properties of spherical and deformed nuclei.

Assuming time reversal invariance, the single-particle Hartree-Fock solutions for axially symmetric deformed nuclei are characterized by the eigenvalue $\Omega_{i}$ of the third component of the total angular momentum on the symmetry axis and by the parity $\pi_{i}$. The state $i$ can be written as

$$
\begin{aligned}
\Phi_{i}(\vec{r}, \sigma, q)= & \chi_{q_{i}}(q)\left[\Phi_{i}^{+}\left(r_{\perp}, z\right) e^{i \Lambda^{-} \varphi} \chi_{+}(\sigma)\right. \\
& \left.+\Phi_{i}^{-}\left(r_{\perp}, z\right) e^{i \Lambda^{+} \varphi} \chi_{-}(\sigma)\right],
\end{aligned}
$$

where $\chi_{q_{i}}(q), \chi_{ \pm}(\sigma)$ are isospin and spin functions, $\Lambda^{ \pm}=$ $\Omega_{i} \pm 1 / 2 \geqslant 0$, and $r_{\perp}, z$, and $\varphi$ are the cylindrical coordinates of $\vec{r}$.

The wave functions $\Phi_{i}$ are expanded into the eigenfunctions $\phi_{\alpha}$ of an axially symmetric deformed harmonic-oscillator potential in cylindrical coordinates. We use 12 major shells in this expansion,

$$
\Phi_{i}(\vec{r}, \sigma, q)=\chi_{q_{i}}(q) \sum_{\alpha} C_{\alpha}^{i} \phi_{\alpha}(\vec{r}, \sigma),
$$

with $\alpha=\left\{n_{\perp}, n_{z}, \Lambda, \Sigma\right\}$ and

$$
\phi_{\alpha}(\vec{r}, \sigma)=\psi_{n_{\perp}}^{\Lambda}\left(r_{\perp}\right) \psi_{n_{z}}(z) \frac{e^{i \Lambda \varphi}}{\sqrt{2 \pi}} \chi_{\Sigma}(\sigma),
$$

in terms of Hermite and Laguerre polynomials

$$
\begin{aligned}
\psi_{n_{z}}(z) & =\sqrt{\frac{1}{\sqrt{\pi} 2^{n_{z}} n_{z} !}} \beta_{z}^{1 / 2} e^{-\xi^{2} / 2} H_{n_{z}}(\xi), \\
\psi_{n_{\perp}}^{\Lambda}\left(r_{\perp}\right) & =\sqrt{\frac{n_{\perp}}{\left(n_{\perp}+\Lambda\right) !}} \beta_{\perp} \sqrt{2} \eta^{\Lambda / 2} e^{-\eta / 2} L_{n_{\perp}}^{\Lambda}(\eta)
\end{aligned}
$$

with

$$
\begin{array}{cl}
\beta_{z}=\left(m \omega_{z} / \hbar\right)^{1 / 2}, & \beta_{\perp}=\left(m \omega_{\perp} / \hbar\right)^{1 / 2}, \\
\xi=z \beta_{z}, & \eta=r_{\perp}^{2} \beta_{\perp}^{2} .
\end{array}
$$


From the expansion (2) we may conveniently express the single-particle Hartree-Fock wave functions in momentum space, which we denote as $\tilde{\Phi}_{i}(\vec{k}, \sigma, q)$. They are given by [48]

$$
\tilde{\Phi}_{i}(\vec{k}, \sigma, q)=\chi_{q_{i}}(q) \sum_{\alpha} C_{\alpha}^{i} \tilde{\phi}_{\alpha}(\vec{k}, \sigma)
$$

with

$$
\tilde{\phi}_{\alpha}(\vec{k}, \sigma)=\frac{1}{(2 \pi)^{3 / 2}} \int d \vec{r} e^{-i \vec{k} \vec{r}} \phi_{\alpha}(\vec{r}, \sigma)
$$

normalized to 1 .

The spin-independent proton, neutron, and total densities are given by

$$
\rho(\vec{r})=\rho\left(r_{\perp}, z\right)=\sum_{i} 2 v_{i}^{2} \rho_{i}\left(r_{\perp}, z\right)
$$

in terms of the occupation probabilities $v_{i}^{2}$ resulting from the BCS equations and the single-particle densities $\rho_{i}$

$$
\rho_{i}(\vec{r})=\rho_{i}\left(r_{\perp}, z\right)=\left|\Phi_{i}^{+}\left(r_{\perp}, z\right)\right|^{2}+\left|\Phi_{i}^{-}\left(r_{\perp}, z\right)\right|^{2},
$$

with

$$
\Phi_{i}^{ \pm}\left(r_{\perp}, z\right)=\frac{1}{\sqrt{2 \pi}} \sum_{\alpha} \delta_{\Sigma, \pm 1 / 2} \delta_{\Lambda, \Lambda^{\mp}} C_{\alpha}^{i} \psi_{n_{\perp}}^{\Lambda}\left(r_{\perp}\right) \psi_{n_{z}}(z) .
$$

Similarly, we define in momentum space the proton, neutron, and total momentum distributions by

$$
n(\vec{k})=n\left(k_{\perp}, k_{z}\right)=\sum_{i} 2 v_{i}^{2} n_{i}\left(k_{\perp}, k_{z}\right),
$$

where $k_{\perp}, k_{z}$ are the cylindrical coordinates of $\vec{k}$. The singleparticle momentum distributions $n_{i}(\vec{k})$ are given by

$$
n_{i}(\vec{k})=n_{i}\left(k_{\perp}, k_{z}\right)=\left|\tilde{\Phi}_{i}^{+}\left(k_{\perp}, k_{z}\right)\right|^{2}+\left|\tilde{\Phi}_{i}^{-}\left(k_{\perp}, k_{z}\right)\right|^{2},
$$

where

$$
\begin{aligned}
\tilde{\Phi}_{i}^{ \pm}\left(k_{\perp}, k_{z}\right)= & \frac{1}{\sqrt{2 \pi}} \sum_{\alpha} \delta_{\Sigma, \pm 1 / 2} \delta_{\Lambda, \Lambda^{\mp}} \\
& \times C_{\alpha}^{i}(-i)^{N} \psi_{n_{\perp}}^{\Lambda}\left(k_{\eta}\right) \psi_{n_{z}}\left(k_{\xi}\right), \\
k_{\xi}= & k_{z} / \beta_{z}, \quad k_{\eta}=k_{\perp}^{2} / \beta_{\perp}^{2}=\frac{k_{x}^{2}+k_{y}^{2}}{\beta_{\perp}^{2}},
\end{aligned}
$$

and $N=\Lambda+n_{z}+2 n_{\perp}$ is the major shell quantum number of the basis state $\alpha$.

The multipole decomposition of the momentum distribution can be written as

$$
\begin{aligned}
n(\vec{k}) & =\sum_{\lambda} n_{\lambda}(k) P_{\lambda}\left(\cos \theta_{k}\right) \\
& =n_{0}(k)+n_{2}(k) P_{2}\left(\cos \theta_{k}\right)+\cdots
\end{aligned}
$$

with multipole components $\lambda$

$$
n_{\lambda}(k)=\frac{2 \lambda+1}{2} \int_{-1}^{+1} P_{\lambda}\left(\cos \theta_{k}\right) n\left(k \cos \theta_{k}, k \sin \theta_{k}\right) d\left(\cos \theta_{k}\right) .
$$

\section{B. Methods going beyond the mean-field approximation}

It is well known (e.g., Ref. [64]) that the methods within the MFA (e.g., shell-model, Hartree-Fock, and others) can describe the nucleon momentum distribution $n(k)$ only for momentum values $k<1.5 \mathrm{fm}^{-1}$ and are unable to explain $n(k)$ for larger $k$. The high-momentum components of $n(k)$ $\left(k>1.5 \mathrm{fm}^{-1}\right)$ are due to the specific forces between the nucleons near the nuclear core $\left(r_{c} \approx 0.4 \mathrm{fm}\right)$ that are the reasons for the short-range and tensor $N N$ correlations. The differences between the values of $n(k)$ for large $k$ obtained within the correlation methods (e.g., $\exp (S)$-method [82], the Jastrow correlation method, and others; for a review see, e.g., Ref. [64]) reach orders of magnitude. In this subsection we consider the effects of $N N$ correlations included in two correlation methods on the high-momentum contributions to the nucleon momentum distribution.

\section{Theoretical approach based on the light-front dynamics method}

Here we derive the NMD obtained within the LFD approach developed in Refs. [61,78]. The latter is based on the nucleon momentum distribution in the deuteron from the light-front dynamics method (e.g., Ref. [79]). Using the natural-orbital representation of the one-body density matrix [83], $n(k)$ is written as a sum of the contributions from the hole-states $\left[\tilde{n}^{h}(k)\right]$ [states up to the Fermi level (F.L.)] and the particlestates $\left[\tilde{n}^{p}(k)\right]$ (see also [61]) for protons $(Z)$ and neutrons $(N)$ :

$$
n_{Z(N)}(k)=\tilde{n}_{Z(N)}^{h}(k)+\tilde{n}_{Z(N)}^{p}(k),
$$

where

$$
\tilde{n}_{Z(N)}^{h}(k)=\frac{C(k)}{Z(N)} \sum_{n l j}^{\text {F.L. }} 2(2 j+1) \lambda_{n l j}\left|\tilde{R}_{n l j}(k)\right|^{2}
$$

and

$$
\tilde{n}_{Z(N)}^{p}(k)=\frac{C(k)}{Z(N)} \sum_{\text {F.L. }}^{\infty} 2(2 j+1) \lambda_{n l j}\left|\tilde{R}_{n l j}(k)\right|^{2} .
$$

In Eqs. (19) and (20)

$$
C(k)=\frac{m_{N}}{(2 \pi)^{3} \sqrt{k^{2}+m_{N}^{2}}},
$$

with $m_{N}$ being the nucleon mass, $\lambda_{n l j}$ the natural occupation numbers, and $\tilde{R}_{n l j}(k)$ the corresponding wave functions in $k$ space of protons (neutrons) in states with quantum numbers $n l j$. The momentum distribution (18) is normalized to unity:

$$
\int n_{Z(N)}(k) d \vec{k}=1 .
$$

As shown in Ref. [78], in the NOR the hole-state contribution $\tilde{n}_{Z(N)}^{h}(k)$ to the momentum distribution can be represented to a good approximation by the momentum distribution from the spherical mean-field methods, i.e., $\lambda_{n l j}$ are taken to be unity and $\tilde{R}_{n l j}(k)$ are replaced by the corresponding mean-field eigenfunctions. In our work we substitute $\tilde{n}_{Z(N)}^{h}(k)$ in Eqs. (18) and (19) by

$$
\tilde{n}_{Z(N)}^{h}(k)=\frac{C(k)}{Z(N)} \tilde{n}_{Z(N)}(k),
$$


where $\tilde{n}_{Z(N)}(k)$ is expressed by the NMD $n_{Z(N)}^{\mathrm{DDHF}}(k)$ obtained within the DDHF formalism (see Sec. II A)

$$
\tilde{n}_{Z(N)}(k)=\frac{Z(N) n_{Z(N)}^{\mathrm{DDHF}}(k)}{\int d \vec{k}^{\prime} C\left(k^{\prime}\right) n_{Z(N)}^{\mathrm{DDHF}}\left(k^{\prime}\right)}
$$

and the normalization is

$$
\int C(k) \tilde{n}_{Z(N)}(k) d \vec{k}=Z(N) .
$$

We note that $\tilde{n}_{Z(N)}^{h}(k), \tilde{n}_{Z(N)}^{p}(k)$, and $\tilde{n}_{Z(N)}(k)$ include the function $C(k)$ that originates from the relativistic LFD approach.

Concerning the particle-state contribution $\left[\tilde{n}_{Z(N)}^{p}(k)\right]$ in Eqs. (18) and (20) we used in Refs. [61,78] the well-known facts that: (i) the high-momentum components of $n(k)$ caused by short-range and tensor correlations are almost completely determined by the contributions of the particle-state natural orbitals (e.g., Ref. [84]) and (ii) the high-momentum tails of the momentum distributions per particle are approximately equal for all nuclei and are a rescaled version of the nucleon momentum distribution in the deuteron $n_{d}(k)$ $[85,86]$,

$$
n_{A}(k) \simeq C^{A} n_{d}(k),
$$

where $C^{A}$ is a constant. These facts made it possible to assume in Refs. [61,78] and later, using the modification of the approach in Ref. [63], that $\tilde{n}_{Z(N)}^{p}(k)$ is related to the high-momentum components of the nucleon momentum distributions in the deuteron. Thus $\tilde{n}_{Z(N)}^{p}(k)$ from Eq. (20) can be substituted by (up to a normalization factor):

$$
\tilde{n}_{Z(N)}^{p}(k)=\beta\left[n_{2}(k)+n_{5}(k)\right],
$$

where $\beta$ is a parameter and $n_{2}(k)$ and $n_{5}(k)$ are expressed by angle-averaged functions [78] as:

$$
n_{2}(k)=C(k) \overline{f_{2}^{2}(k)}
$$

and

$$
n_{5}(k)=C(k) \overline{\left(1-z^{2}\right) f_{5}^{2}(k)} \text {. }
$$

In Ref. (29) $z=\cos (\widehat{\vec{k}, \vec{n}}), \vec{n}$ is a unit vector along the threevector $(\vec{\omega})$ component of the four-vector $\omega$ that determines the position of the light-front surface [79]. The functions $f_{2}(k)$ and $f_{5}(k)$ are two of the six scalar functions $f_{1-6}\left(k^{2}, \vec{n} \cdot \vec{k}\right)$ that are the components of the deuteron total wave function $\Psi(\vec{k}, \vec{n})$. It was shown [79] that $f_{5}$ largely exceeds other $f$ components for $k \geqslant 2.0-2.5 \mathrm{fm}^{-1}$ and is the main contribution to the high-momentum component of $n_{d}(k)$, incorporating the main part of the short-range properties of the $N N$ interaction. It was shown in Ref. [63], however, that not only $n_{5}(k)$ [originating from $f_{5}(k)(29)$ ] but also $n_{2}(k)$ [related to $f_{2}(k)(28)$ ] has to be included partially in the particle-state contribution $\tilde{n}_{Z(N)}^{p}(k)$ to the momentum distribution. The latter leads to a successful explanation of the experimental data for the quasielastic scaling function $f^{Q E}\left(\psi^{\prime}\right)$ (see Fig. 4 of Ref. [63]) extracted from inclusive electron scattering off nuclei.
Finally, the normalized to unity proton (neutron) momentum distribution [Eqs. (18)-(22)] has the form:

$$
n_{Z(N)}(k)=\frac{C(k) \tilde{n}_{Z(N)}(k)+Z(N) \beta\left[n_{2}(k)+n_{5}(k)\right]}{\int d \vec{k}^{\prime}\left\{C\left(k^{\prime}\right) \tilde{n}_{Z(N)}\left(k^{\prime}\right)+Z(N) \beta\left[n_{2}\left(k^{\prime}\right)+n_{5}\left(k^{\prime}\right)\right]\right\}}
$$

[with normalization of $\tilde{n}_{Z(N)}(k)$ presented by Eq. (25)].

For the value of the parameter $\beta$ we choose $\beta=5.0$ because of three reasons. First, our calculations of $n_{Z(N)}(k)$ for various nuclei using this value lead to results very close to the empirical data for the nucleon momentum distribution $n_{\mathrm{CW}}$ (see Fig. 2 of Ref. [61] and Fig. 3 of Ref. [63]) extracted in Refs. [55,87] from the $y$-scaling analyses of inclusive electron scattering off nuclei. Second, our estimations of the high-momentum components of $n_{Z(N)}(k)$ [Eq. (30)] showed that the value of $\beta$ must be similar to the value of $C^{A}$ [Eq. (26)]. It is shown (see Table I in Ref. [86]) that the value of $C^{A}$ estimated from the height of the plateau exhibited by the ratio of the nucleon momentum distribution of a nucleus to the one of the deuteron at $k>2.0 \mathrm{fm}^{-1}$ ranges from 4.0 for ${ }^{12} \mathrm{C}$ to 4.4 for ${ }^{40} \mathrm{Ca}, 4.5$ for ${ }^{56} \mathrm{Fe}, 4.8$ for ${ }^{208} \mathrm{~Pb}$, and 4.9 for nuclear matter $(A=\infty)$. Third, our results for the NMD (e.g., for ${ }^{12} \mathrm{C},{ }^{64} \mathrm{Ni}$, and others) in LFD approach for large values of $k\left(k>2.0 \mathrm{fm}^{-1}\right)$ are similar to those obtained in the local density approximation using the Jastrow correlation method [80].

\section{Theoretical approach based on the local density approximation}

It is well known that the inclusion of correlations in nuclear matter modifies the occupation probability predicted by the local density Fermi gas model. It was shown in Ref. [80] that for a finite nucleus the separation between the meanfield contribution and correlation effects can be performed in an analogous way. According to Ref. [80] one can introduce proton (neutron) momentum distribution in a general way:

$$
n_{Z(N)}(k)=n_{Z(N)}^{M F A}(k)+\delta n_{Z(N)}(k),
$$

where $n_{Z(N)}^{M F A}(k)$ is the mean-field contribution that can be momentum distribution corresponding to a Slater determinant generated by the single-particle wave functions in the momentum space or HF momentum distribution, while $\delta n_{Z(N)}(k)$ embodies the corrections due to dynamical correlations not included in the MFA. If one applies the LDA to the second term of Eq. (31), the nucleon momentum distribution $n_{Z(N)}(k)$ can be written in the form:

$$
n_{Z(N)}(k)=n_{Z(N)}^{M F A}(k)+\frac{1}{4 \pi^{3}} \int \delta v\left[k_{F}^{Z(N)}(r), k\right] d \vec{r},
$$

where $\delta v\left[k_{F}^{Z(N)}(r), k\right]$ corresponds to the occupation probability that is entirely due to the effects of dynamical correlations induced by the $N N$ interaction. The local Fermi momentum $k_{F}^{Z(N)}(r)$ is related to the proton (neutron) density through the relation

$$
k_{F}^{Z(N)}(r)=\left[3 \pi^{2} \rho_{Z(N)}(r)\right]^{1 / 3} .
$$

By definition of $k_{F}^{Z(N)}(r)$ one has $\int \delta v\left[k_{F}^{Z(N)}(r), k\right] d \vec{k}=0$. For convenience, a phenomenological procedure based on 
the results of the lowest-order cluster (LOC) approximation developed in Ref. [88] has been followed to evaluate explicitly the correlated term. Choosing a correlation function of the form

$$
f(r)=1-e^{-\gamma^{2} r^{2}},
$$

the LOC gives for $\delta v\left[k_{F}^{Z(N)}, k\right][80]$

$$
\begin{aligned}
\delta v\left[k_{F}^{Z(N)}(r), k\right]= & {\left[Y(k, 8)-k_{\mathrm{dir}}\right] \Theta\left[k_{F}^{Z(N)}(r)-k\right] } \\
& +8\left\{k_{\mathrm{dir}} Y(k, 2)-[Y(k, 4)]^{2}\right\},
\end{aligned}
$$

where

$$
\begin{aligned}
c_{\mu}^{-1} Y(k, \mu)= & \frac{e^{-\tilde{k}_{+}^{2}}-e^{-\tilde{k}_{-}^{2}}}{2 \tilde{k}^{2}}+\int_{0}^{\tilde{k}_{+}} e^{-y^{2}} d y \\
& +\operatorname{sgn}\left(\tilde{k}_{-}\right) \int_{0}^{\left|\tilde{k}_{-}\right|} e^{-y^{2}} d y
\end{aligned}
$$

with

$$
\begin{aligned}
& c_{\mu}=\frac{1}{8 \sqrt{\pi}}\left(\frac{\mu}{2}\right)^{3 / 2}, \quad \tilde{k}=\frac{k}{\gamma \sqrt{\mu}}, \\
& \tilde{k}_{ \pm}=\frac{k_{F} \pm k}{\gamma \sqrt{\mu}}, \quad \operatorname{sgn}(x)=\frac{x}{|x|} .
\end{aligned}
$$

The quantity

$$
\begin{aligned}
k_{\mathrm{dir}} & =\frac{2\left[k_{F}^{Z(N)}\right]^{3}}{3 \pi^{2}} \int[f(r)-1]^{2} d \vec{r} \\
& =\frac{1}{3 \sqrt{2 \pi}}\left[\frac{k_{F}^{Z(N)}}{\gamma}\right]^{3}
\end{aligned}
$$

is the direct part of the Jastrow wound parameter.

As in the approach based on the LFD method discussed before, in our work we use for $n_{Z(N)}^{\mathrm{MFA}}(k)$ the momentum distributions obtained from DDHF calculations $n_{Z(N)}^{\mathrm{DDHF}}(k)$ with normalization given by:

$$
\int n_{Z(N)}^{\mathrm{DDHF}}(k) d \vec{k}=Z(N) .
$$

For the densities $\rho_{Z(N)}(r)$ entering Eq. (33) we use the $\mathrm{HF}+\mathrm{BCS}$ proton (neutron) densities [53] normalized as:

$$
\int \rho_{Z(N)}^{\mathrm{DDHF}}(r) d \vec{r}=Z(N) .
$$

For the correlation factor in Eq. (34) we adopt the same value $\gamma=1.1 \mathrm{fm}^{-1}$ as in Ref. [80] which is taken from the microscopic nuclear matter calculations [89] but seems to agree also with the data on momentum distributions $n(k)$ in finite nuclei.

\section{RESULTS OF CALCULATIONS AND DISCUSSION}

We start by showing the results of our calculations for the monopole components of $n(\vec{k})$ and $\rho(\vec{r})$ in the ${ }^{100,120,136} \mathrm{Sn}$ isotopes. As discussed in Refs. [48,53] these are the only important components for the HF momentum distributions $\left(n(\vec{k}) \cong n_{0}(\vec{k}) \equiv n(k)[\right.$ Eq. (16)] $)$. For easier reading, in the figures we will omit the subscript $Z(N)$ indices of $n(k)$ for the proton and neutron momentum distributions replacing them by $p(n)$, respectively. In Fig. 1(a) are given the total momentum distributions for ${ }^{100} \mathrm{Sn},{ }^{120} \mathrm{Sn}$, and ${ }^{136} \mathrm{Sn}$ plotted in nonlogarithmic scale. One can observe in the figure an appreciable difference between the curves in the low-momentum region and, at the same time on this scale, no sensitivity of $n(k)$ at $k>1 \mathrm{fm}^{-1}$ when increasing the number of neutrons. As can be expected, the momentum distributions calculated within the DDHF approach show a steep decrease up to $k \leqslant 2 \mathrm{fm}^{-1}$ that can be seen in all mean-field calculations. Here we emphasize that the effects of $N N$ correlations on the momentum distributions can be seen at higher momenta $\left(k \geqslant 2 \mathrm{fm}^{-1}\right)$ when we will compare the DDHF results with those obtained in the approaches that take them into account. The results for $n(k)$ are related with the total density profiles of the selected three $\mathrm{Sn}$ isotopes shown in Fig. 1(b). For a comparison, in the same figure we present the total density $\rho(r)$ of ${ }^{100} \mathrm{Sn}$ obtained in Ref. [90] within the Hartree-FockBogoliubov model using the SLy4 parametrization of the Skyrme force. We note, following the discussion concerning Fig. 5 in Ref. [53], that the effect of adding more and more neutrons leads to an extension of the total density $\rho(r)$ as one goes from ${ }^{100} \mathrm{Sn}$ to ${ }^{136} \mathrm{Sn}$ and to an emergence of a region at the nuclear surface quantified as a "neutron skin." The latter is due to the larger spatial extension of the neutron density relatively to the proton one. The same consistent trends of the matter densities when increasing the mass number $A$ were found in Ref. [90]. One can see from Fig. 1(b) that the densities of ${ }^{100} \mathrm{Sn}$ obtained in our previous work [53] and in Ref. [90] are very similar. Second, the same choice of two extreme neutron-deficient and neutron-rich isotopes and one stable isotope between them in the Sn chain [53] makes the difference in the results for $n(k)$ at low $k$ more pronounced with respect to those for $\rho(r)$ at large $r$.

The effect of pairing correlations on the momentum distribution can be seen in Fig. 2. We restrict the discussion to the stable ${ }^{84} \mathrm{Kr}$ isotope that is weakly deformed. In this case the pairing correlations have been included by solving the BCS equations in the constant pairing gap mode. Figure 2 shows that the effect of pairing correlations is small. At high $k$ this effect plays some role resulting in more pronounced tails when BCS correlations are included in the calculations. Nevertheless, as we will illustrate and discuss later, the effects of pairing correlations on the HF momentum distribution of nuclear matter and of finite nuclei are very different being much larger in the case of NM. Because there is no big difference among the results for $n_{p}(k), n_{n}(k)$, and $n(k)$ with or without BCS correlations included, afterwards we will use in our consideration only the results from the complete DDHF + BCS calculations.

Our next step is to present and discuss the results for the NMD's of exotic nuclei obtained also in correlation methods. A comparison of these results for the neutron and proton momentum distributions of ${ }^{64} \mathrm{Ni},{ }^{84} \mathrm{Kr}$, and ${ }^{120} \mathrm{Sn}$ nuclei is given in Fig. 3 together with the HF momentum distributions. As can be seen, for all nuclei the inclusion of $N N$ correlations strongly affects the high-momentum region of NMD. At $k>$ $1.5 \mathrm{fm}^{-1}$ both LFD and LDA momentum distributions start to deviate from the DDHF + BCS case. They behave rather 

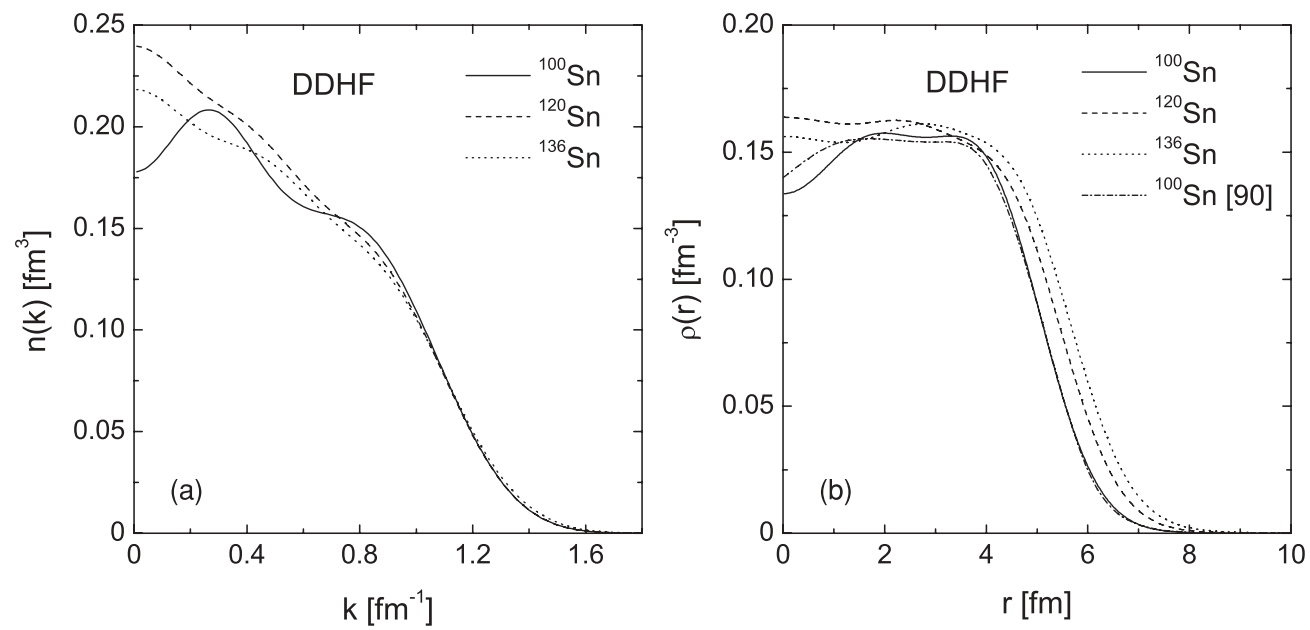

FIG. 1. (a) Total momentum distribution for ${ }^{100} \mathrm{Sn},{ }^{120} \mathrm{Sn}$, and ${ }^{136} \mathrm{Sn}$ isotopes resulting from DDHF calculations. The normalization is $\int n(k) d \vec{k}=1$; (b) total density distributions for the same $\mathrm{Sn}$ isotopes. The normalization is $\int \rho(r) d \vec{r}=A$.

similarly in the interval $1.5<k<3 \mathrm{fm}^{-1}$. At $k>3 \mathrm{fm}^{-1}$ the LFD method predicts systematically higher momentum components compared to LDA momentum distributions. This observation can be explained by the different extent to which $N N$ correlations are taken into account in both approaches. Our results for the NMD's in the LFD method for large values of $k\left(k>2 \mathrm{fm}^{-1}\right)$ are similar to those obtained within the Jastrow correlation method and, thus, the high-momentum tails of $n(k)$ are caused by the short-range $N N$ correlations. The LDA approach through the nuclear matter dynamic effects and using the local Fermi momentum $k_{F}^{Z(N)}(r)$ calculated selfconsistently by means of the HF density [Eq. (33)] produces less pronounced high-momentum tail, yet even so the results are very close to those obtained in the LFD method. As was already shown, at $k>1.5 \mathrm{fm}^{-1}$ the DDHF $+\mathrm{HF}$ momentum distributions fall off rapidly by several orders of magnitude in contrast to the correlated NMD's. In addition, we observe that (i) the results shown above are similar for all nuclei in a given isotopic chain and going from $\mathrm{Ni}$ to $\mathrm{Sn}$ isotopes, as well; (ii) the behavior of $n(k)$ is similar for protons and neutrons; (iii) at high $k$ the proton and neutron NMD's obtained within the LFD method cannot be distinguished from each other because the high-momentum tails in this approach are determined by the high-momentum component of the nucleons in the deuteron [78]; (iv) concerning the NMD's calculated in the LDA approach, some difference between $n(k)$ for protons
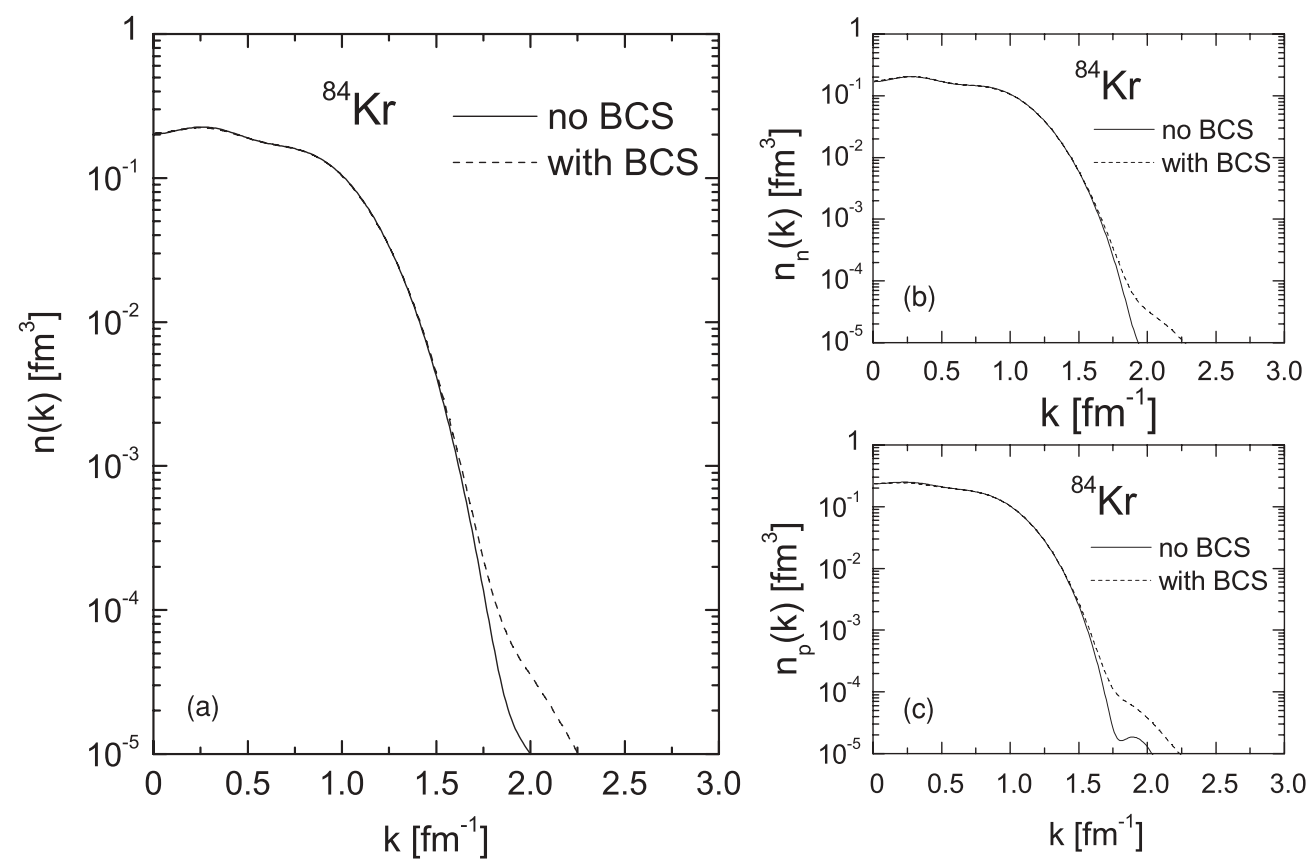

FIG. 2. DDHF results for the total (a), neutron (b), and proton (c) momentum distributions with (dashed line) and without (solid line) pairing for ${ }^{84} \mathrm{Kr}$. The normalization is $\int n_{n(p)}(k) d \vec{k}=\int n(k) d \vec{k}=1$. 


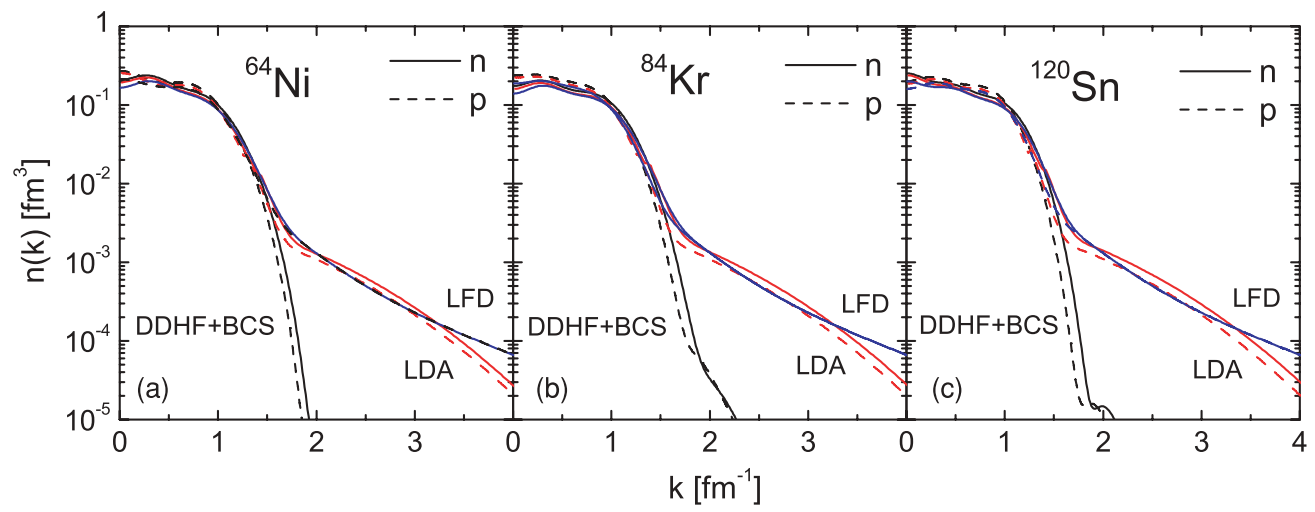

FIG. 3. (Color online) Neutron (solid line) and proton (dashed line) momentum distributions obtained within the DDHF + BCS (black), LFD (blue), and LDA (red) methods for ${ }^{64} \mathrm{Ni}(\mathrm{a}),{ }^{84} \mathrm{Kr}(\mathrm{b})$, and ${ }^{120} \mathrm{Sn}(\mathrm{c})$ nuclei. The normalization is $\int n_{n(p)}(k) d \vec{k}=1$.

and neutrons can be observed due to $Z(N)$ dependence of the local Fermi momentum $k_{F}$.

In Figs. 4-7 we show the neutron $n_{n}(k)$ (Figs. 4 and 6) and proton $n_{p}(k)$ (Figs. 5 and 7) momentum distributions of some selected isotopes in the $\mathrm{Ni}$ and $\mathrm{Sn}$ chains, the same that have been considered in Ref. [53] to calculate important nuclear properties in coordinate space. The results are presented in both logarithmic and linear scales to study the isotopic sensitivity of these momentum distributions in the high-momentum region and in the region of small momenta, respectively. In addition, in each of the figures the results for neutron and proton momentum distributions in the DDHF + BCS method and in the correlation LFD and LDA approaches at $k<2 \mathrm{fm}^{-1}$ are given separately in panels (b) and (c). First, it is seen from the figures that the general trend in the behavior of NMD's obtained within the methods used in the calculations and already shown in Fig. 3 is preserved. Second, the evolution of the NMD's as we increase the number of neutrons consists of an increase of the high-momentum tails (for $k>1.5 \mathrm{fm}^{-1}$ ) of $n_{n}(k)$, while the effect on $n_{p}(k)$ is opposite. However, the spreading of the tails corresponding to $n_{p}(k)$ of the considered isotopes is of the same order although the number of protons remains the same. In this respect, the results presented in Figs. 5 and 7 are challenging because they show how proton momentum distributions "feel" the different number of neutrons in exotic nuclei. We also emphasize that the LFD method does not show this isotopic sensitivity, in contrast to the HF and LDA methods that still demonstrate this trend. Concerning the low-momentum region it can be seen from Figs. 4-7 that NMD's are very sensitive to the details of the calculations. In this region $n_{n}(k)$ decreases while, on the contrary, $n_{p}(k)$ increases with the increase of the number
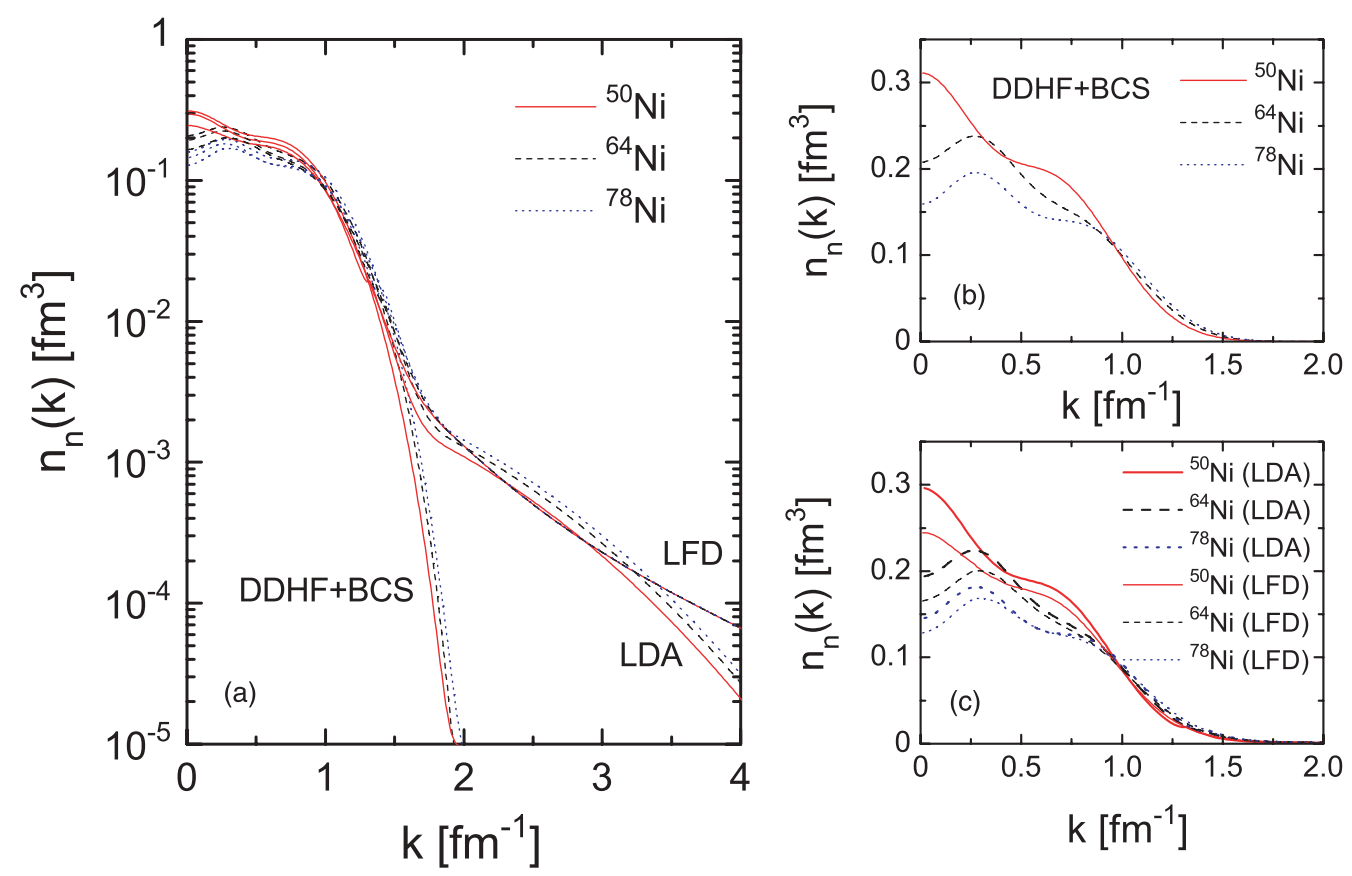

FIG. 4. (Color online) (a) Neutron momentum distributions obtained within the DDHF + BCS, LFD, and LDA methods for ${ }^{50} \mathrm{Ni}$ (solid line), ${ }^{64} \mathrm{Ni}$ (dashed line), and ${ }^{78} \mathrm{Ni}$ (dotted line) isotopes. The normalization is $\int n_{n}(k) d \vec{k}=1$. The DDHF + BCS results, as well as the LFD and LDA results, are separately shown in a linear scale in (b) and (c), respectively. 

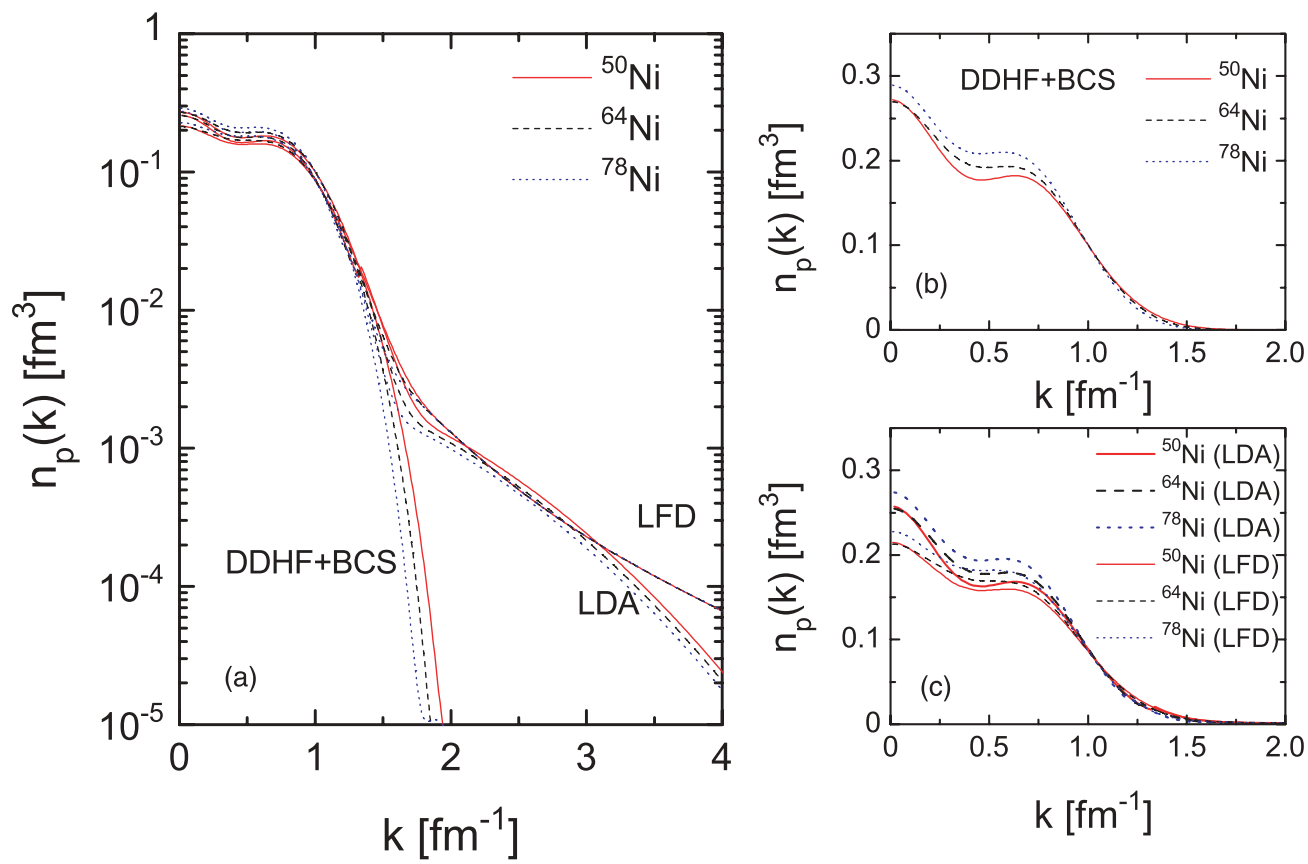

FIG. 5. (Color online) The same as in Fig. 4 but for the proton momentum distributions.

of neutrons $N$. This is a common feature of the calculated results obtained in all methods. Nevertheless, in this region the spreading is considerably reduced. From the comparison of the proton and neutron momentum distributions at low momenta it comes out that the protons exhibit the same trend [Figs. 5(b), 5(c), 7(b), and 7(c)], while the neutrons are more sensitive to nuclear shell effects (see panels (b) and (c) of Figs. 4 and 6).

The isotonic sensitivity of the neutron, proton, and total momentum distributions of ${ }^{78} \mathrm{Ni},{ }^{86} \mathrm{Kr}$, and ${ }^{100} \mathrm{Sn}$ nuclei $(N=$
50) is shown in Fig. 8. It can be seen from the figure a small difference between the different curves when using a given approach (DDHF + BCS or LDA). The neutron momentum distributions $n_{n}(k)$ do not differ so much in comparison to the proton momentum distributions $n_{p}(k)$. The relative contributions of $n_{n}(k)$ and $n_{p}(k)$ to the total momentum distribution $n(k)$ lead to almost equal high-momentum tails of $n(k)$ for these isotones. This is in accordance with the well-known results showing that the tails of $n(k) / A$ are almost equal for all nuclei (for a review, see, e.g., Ref. [64]).
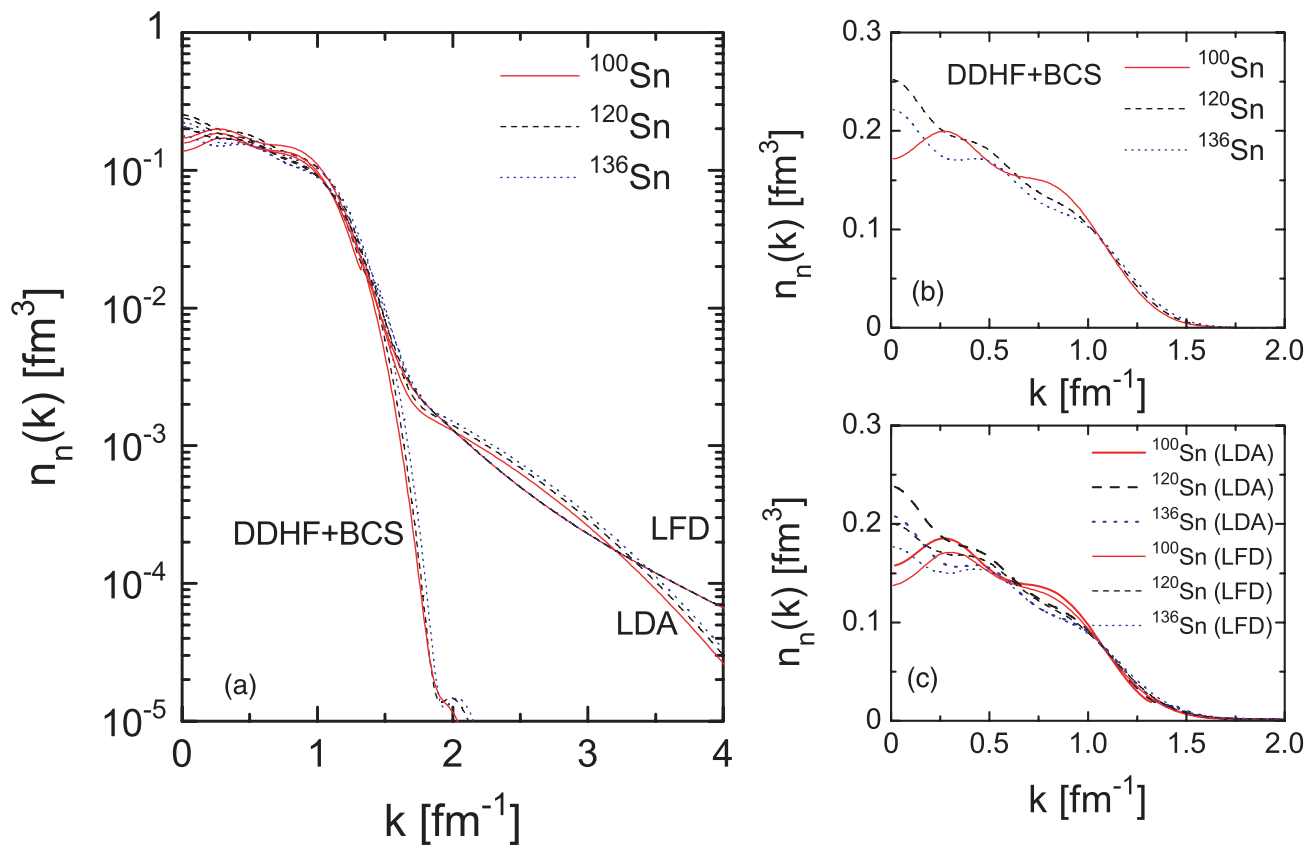

FIG. 6. (Color online) The same as in Fig. 4 but for the ${ }^{100} \mathrm{Sn}$ (solid line), ${ }^{120} \mathrm{Sn}$ (dashed line), and ${ }^{136} \mathrm{Sn}$ (dotted line) isotopes. 

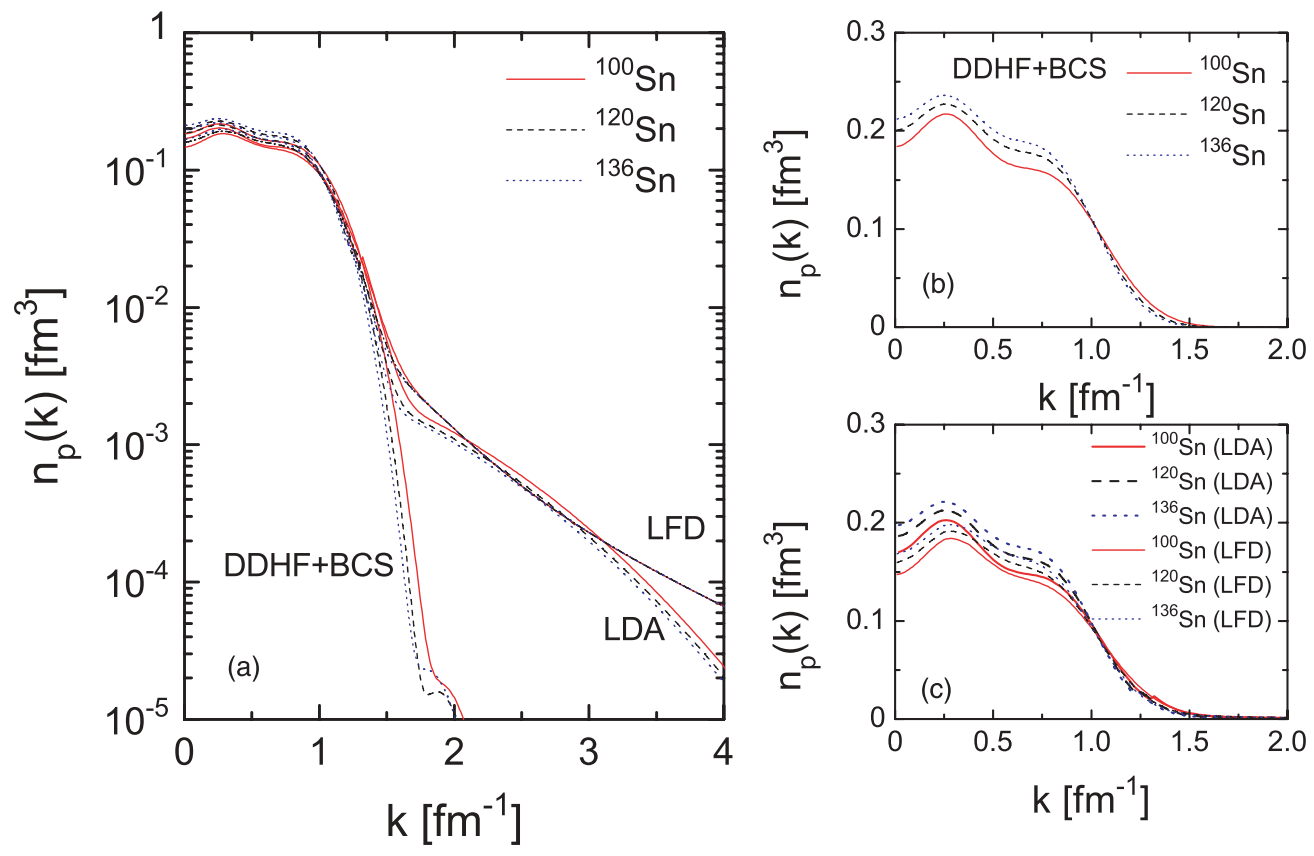

FIG. 7. (Color online) The same as in Fig. 6 but for the proton momentum distributions.

The role of the deformation of the mean field on the NMD is studied on the example of ${ }^{98} \mathrm{Kr}$ isotope. For this purpose, we show in Fig. 9 the intrinsic momentum distribution for neutrons and protons and for oblate and prolate shapes of ${ }^{98} \mathrm{Kr}$. The results of the three methods considered in our work are given and compared together with the result for the spherical case obtained within the HF method. The differences between the momentum distributions calculated within a given theoretical method are negligible (especially at high $k$ ) and practically cannot be distinguished. Thus, we find almost no dependence of the $n_{n}(k)$ and $n_{p}(k)$ on the character of deformation.

The effects of the correlations included in the LFD and LDA methods on the neutron momentum distribution of ${ }^{120} \mathrm{Sn}$ are presented in Fig. 10(a). The parameters that govern the correlations in the two approaches are $\beta$ and $\gamma$, respectively. The results of the calculations for three values of each of them are shown by thick lines in the case of LDA and by thin lines in the case of LFD method. The value $\beta=5$ is the upper limit of this parameter [86] and, therefore, we give in Fig. 10(a) also the results for $n_{n}(k)$ for two smaller values $\beta=4.4$ and $\beta=4.7$. It turns out that $n_{n}(k)$ does not change significantly for different values of $\beta$, thus showing the strong presence of correlations at short distances within the LFD method not only for the stable but also for the exotic nuclei. However, a larger sensitivity of $n_{n}(k)$ on the parameter $\gamma$ in the LDA approach appears, particularly in the interval $1.5<k<3 \mathrm{fm}^{-1}$. The

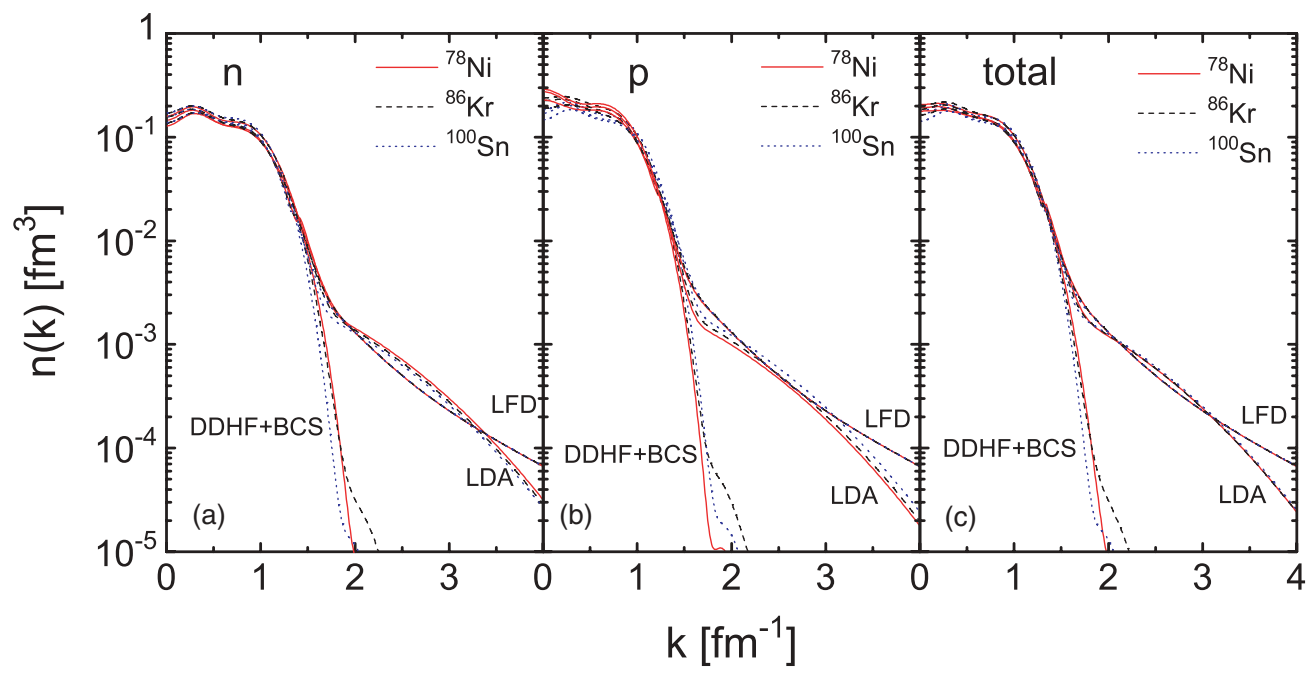

FIG. 8. (Color online) Neutron (a), proton (b), and total (c) momentum distributions obtained within the DDHF + BCS, LFD, and LDA methods for ${ }^{78} \mathrm{Ni}$ (solid line), ${ }^{86} \mathrm{Kr}$ (dashed line), and ${ }^{100} \mathrm{Sn}$ (dotted line) isotones. The normalization is $\int n_{n(p)}(k) d \vec{k}=\int n(k) d \vec{k}=1$. 

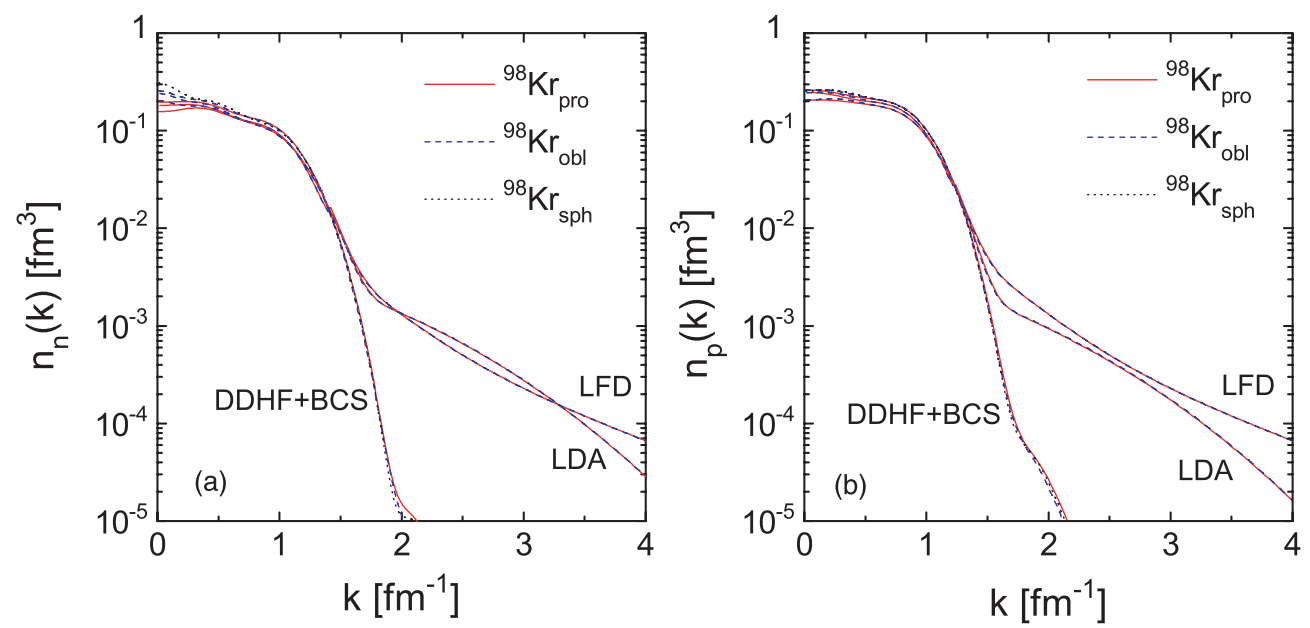

FIG. 9. (Color online) Neutron (a) and proton (b) momentum distributions obtained within the DDHF + BCS, LFD, and LDA methods corresponding to oblate (dashed line) and prolate (solid line) shape of ${ }^{98} \mathrm{Kr}$. For comparison, the spherical case (dotted line) within the $\mathrm{DDHF}+\mathrm{BCS}$ method is also given. The normalization is $\int n_{n(p)}(k) d \vec{k}=1$.

value $\gamma=1.1 \mathrm{fm}^{-1}$ (from Ref. [80]) and two more values $\gamma=0.9 \mathrm{fm}^{-1}$ and $\gamma=1.3 \mathrm{fm}^{-1}$ are used in the calculations. As expected, the obtained neutron momentum distributions contain smaller correlation effects at larger values of $\gamma$. This is in accordance with the behavior of the correlation function $f(r)$ [see Eq. (34)]. At $k>3 \mathrm{fm}^{-1}$ the LDA results start to deviate in an opposite way, but in this very highmomentum region no definite conclusion about the role of correlations can be drawn.

In our work we study also the sensitivity of our results for NMD to different effective $N N$ forces. In Fig. 10(b) we show the neutron momentum distributions of ${ }^{120} \mathrm{Sn}$ obtained by using SLy4 Skyrme force together with the results obtained from other parametrizations, namely Sk3 [91] and SG2 [92]. For stable spherical nuclei it is known that all the Skyrme-type effective interactions give similar results for the total momentum distribution [93]. We would like to resolve possible ambiguities concerning exotic nuclei by comparing the momentum distributions for neutrons on the example of the stable ${ }^{120} \mathrm{Sn}$ isotope. In fact, some sensitivity to different Skyrme forces can be observed in the inset of Fig. 10(b) for momenta $k<0.5 \mathrm{fm}^{-1}$. This is due to shell effects that are
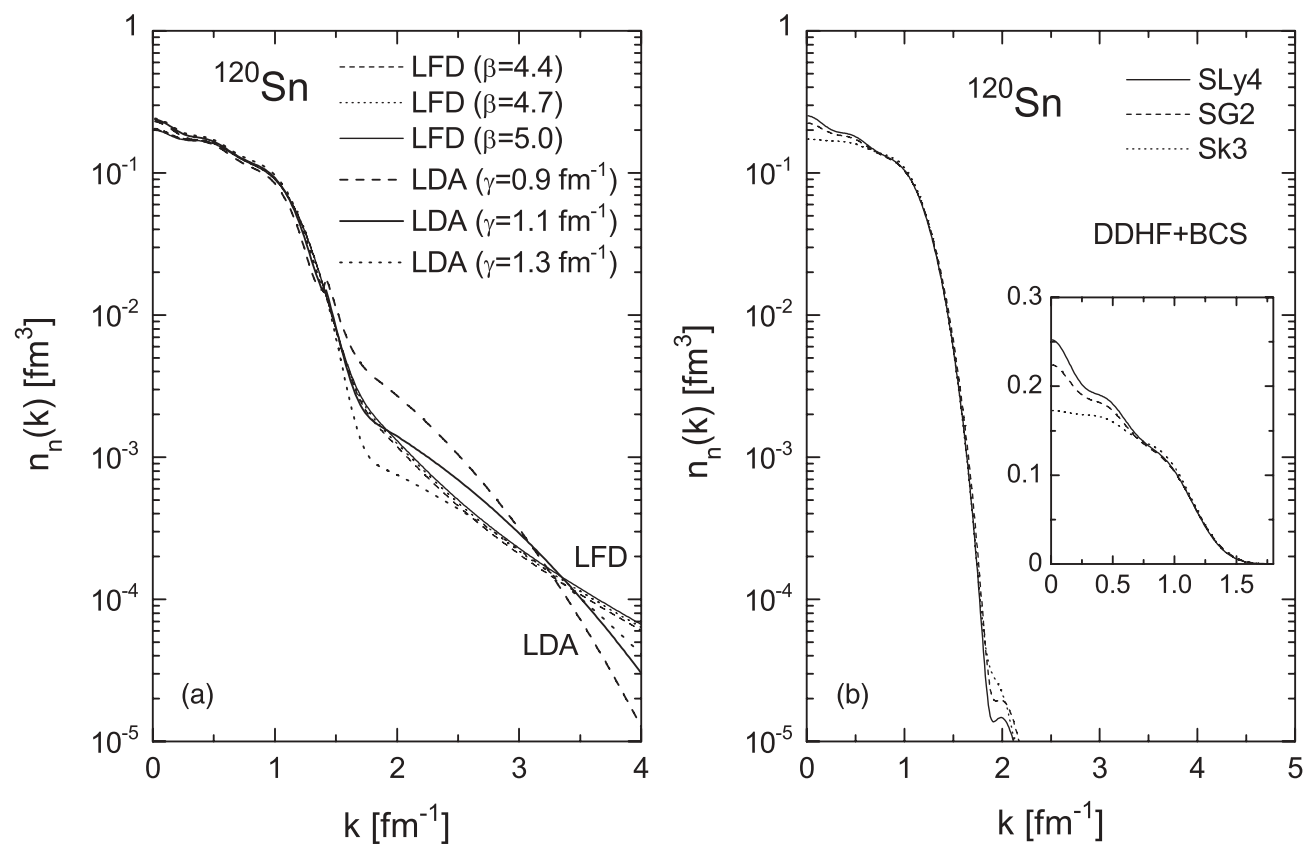

FIG. 10. (a) Neutron momentum distributions obtained within the LFD (for different values of the parameter $\beta$ ) and LDA (for different values of the parameter $\gamma$ ) methods for ${ }^{120} \mathrm{Sn}$; (b) neutron momentum distributions obtained within the DDHF $+\mathrm{BCS}$ method for ${ }^{120} \mathrm{Sn}$ and for different Skyrme forces. The normalization is $\int n_{n}(k) d \vec{k}=1$. 


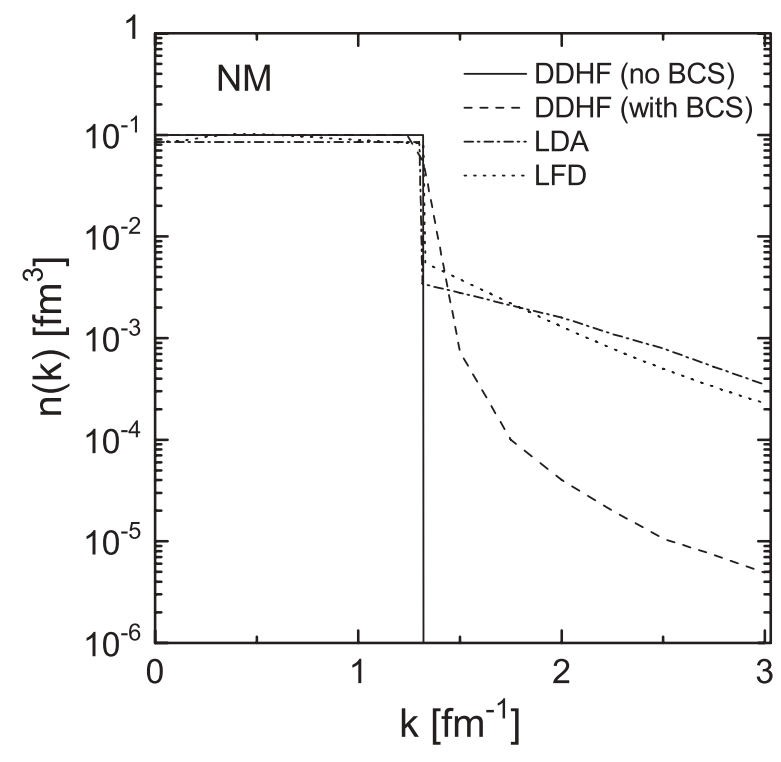

FIG. 11. Comparison of DDHF results for the momentum distributions of nuclear matter with (dashed line) and without (solid line) pairing correlations with the results from LFD (dotted line) and LDA (dash-dotted line) methods.

found also in the density profiles of the nucleus. Generally, as it has been pointed out in Ref. [53], the predictions for the charge and matter rms radii do not differ too much when different Skyrme forces are used. In analogy, different Skyrme interactions also produce similar results for the NMD.

Finally, in Fig. 11 a comparison of the results for the total momentum distribution $n(k)$ of nuclear matter calculated within the MFA and correlation methods used in our work is shown. The HF momentum distribution is strongly affected by pairing correlations that build up a long tail at high momentum $\left(k>k_{F}\right)$. Comparing this result with the result illustrated in Fig. 2 for ${ }^{84} \mathrm{Kr}$, the different role played by pairing correlations on the DDHF momentum distribution of NM and of finite nuclei becomes clear. Moreover, it is interesting to explore the case when one includes other type of correlations in NM. Stringari et al. [80] have already shown in their model based on the LDA the prediction for $n(k)$ in the case of nuclear matter. An enhancement of the high-momentum components of $n(k)$ can be seen from Fig. 11 when both LDA and LFD methods are used. Hence, in nuclear matter the effects of short-range and tensor correlations are much stronger than the BCS correlations taken into account in the DDHF + BCS calculations.

\section{CONCLUSIONS}

In this work we investigated the properties of the momentum distributions of medium and heavy exotic nuclei, especially of $\mathrm{Ni}, \mathrm{Kr}$, and Sn even-even isotopes. The theoretical study was performed on the base of the mean-field method, as well as of two correlation methods taking into account the $N N$ correlations at short distances. The neutron, proton, and total momentum distributions of these nuclei were calculated within (i) a deformed DDHF + BCS method with Skyrmetype effective interactions [47,48], (ii) a theoretical approach
[61,63,78] based on the light-front dynamics method [79], and (iii) a theoretical model based on the local density approximation [80]. In the DDHF + BCS calculations we consider the monopole component of $n(\vec{k})\left(n(\vec{k}) \cong n_{0}(\vec{k})\right)$ because this is the only important component in the expansion of the HF ground-state momentum distribution [Eq. (16)]. However, the two correlation approaches allow one to include both the mean-field and short-range effects for the description of the nucleon momentum distribution.

The study of the isotopic sensitivity of various kinds of momentum distributions shows different trends. For a given isotopic chain, we find that in the high-momentum region $(k>$ $1.5 \mathrm{fm}^{-1}$ ) the high-momentum tails of the neutron momentum distributions $n_{n}(k)$ increase with the increase of the number of neutrons $N$, whereas the proton momentum distributions $n_{p}(k)$ exhibit an opposite effect. In the same region the LFD method does not show this isotopic sensitivity, in contrast to the DDHF + BCS and LDA methods. At low momenta $n_{n}(k)$ decreases while, on the contrary, $n_{p}(k)$ increases with the increase of $N$. Additionally to the isotopic sensitivity we studied how the momentum distributions of some isotones are modified keeping the neutron number constant. We find that the total momentum distributions of ${ }^{78} \mathrm{Ni},{ }^{86} \mathrm{Kr}$, and ${ }^{100} \mathrm{Sn}$ nuclei $(N=$ 50) reveal the same high-momentum tails in all methods used.

The role of the deformation on the momentum distributions is discussed in the present work on the example of ${ }^{98} \mathrm{Kr}$ isotope. As it has been found in Refs. [48,94], the isotropy of the total momentum distribution is a property of the nucleus at equilibrium. Our results for the neutron and proton momentum distributions of this nucleus show small changes in the overall behavior for the oblate and prolate shapes. Although the neutron and proton densities change with deformation [53], the momentum distributions demonstrate a very weak dependence on the character of deformation. This is valid for all three theoretical approaches explored in our work.

The pairing correlations are shown to influence the highmomentum behavior of the neutron $n_{n}(k)$, proton $n_{p}(k)$, and total $n(k)$ momentum distributions in the case of ${ }^{84} \mathrm{Kr}$, but the differences between the results with or without BCS correlations included in the calculations are very small. The latter are even smaller considering nuclei from the $\mathrm{Ni}$ and Sn isotopic chains that have a spherical shape. The effect of pairing correlations on the HF momentum distribution is much stronger in the case of nuclear matter producing a tail for momenta $k>k_{F}$.

The effects of the dynamical correlations on the momentum distributions have been calculated using approaches that account for correlations at short distances. In the correlation approach based on LFD, the incorporation of the LFD result for the nucleon momentum distribution in the deuteron makes it possible to study NMD's also for exotic nuclei, especially at high momenta $\left(k \geqslant 2 \mathrm{fm}^{-1}\right)$. It is known $[85,86]$ that the latter are rescaled versions of $n(k)$ in the deuteron. This is the main reason for the fact that the LFD calculations do not show isotopic sensitivity on the obtained NMD's. Concerning the NMD's calculated in the LDA approach in which a Jastrow-type correlation function is adopted, some difference between $n(k)$ for protons and neutrons is observed due to $Z(N)$ dependence of the local Fermi momentum $k_{F}$. In 
general, a strong enhancement of the momentum distributions at large $k$ is observed in comparison to the MFA result and, at the same time, both LFD and LDA results are similar in the high-momentum region. In our work we pay a particular attention to the dependence of the results for the momentum distributions on the correlation parameters $\beta$ and $\gamma$. In the LFD calculations we used the value of the parameter $\beta=5.0$. It turned out that this value leads to a good agreement with the empirical data for $n(k)$ extracted from the $y$-scaling analyses of inclusive electron scattering off nuclei $[55,87]$. The LDA calculations have been carried out for $\gamma=1.1 \mathrm{fm}^{-1}$, the same value being adopted in nuclear matter and also providing a good choice for finite nuclei. In addition, we note that our prediction for $n(k)$ in NM obtained within the LFD method is in agreement with the LDA result [80]. In our opinion, however, the question for the specific values of the parameters $\beta$ and $\gamma$ that determine the strength of the correlations is still open.

We emphasize that, in our work, a possible practical way to make predictions for the momentum distributions of exotic nuclei far from the stability line is proposed that provides a systematic description of $n(k)$ in medium-weight and heavy nuclei. The comparison of the predicted nucleon momentum distributions with the results of possible experiments using a colliding electron-exotic nuclei storage rings would show the effect of the neutron excess in these nuclei and will be also a test of various theoretical models of the structure of exotic nuclei.

\section{ACKNOWLEDGMENTS}

This work was partly supported by the Bulgarian National Science Fund under Contract No. 02-285, by Ministerio de Ciencia e Innovación (Spain) under Contract Nos. FIS200801301 and FPA2007-62616, and by the Agreement between CSIC (Spain) and the Bulgarian Academy of Sciences (2007BG0011). One of the authors (M.V.I.) acknowledges the support from the European Operational Program HRD through Contract BGO051PO001/07/3.3-02/53 with the Bulgarian Ministry of Education, Youth and Science.
[1] I. Tanihata, H. Hamagaki, O. Hashimoto, S. Nagamiya, Y. Shida, H. Yoshikawa, O. Yamakawa, K. Sugimoto, T. Kobayashi, D. E. Greiner, N. Takahashi, and Y. Nojiri, Phys. Lett. B160, 380 (1985).

[2] I. Tanihata, H. Hamagaki, O. Hashimoto, Y. Shida, N. Yoshikawa, K. Sugimoto, O. Yamakawa, T. Kobayashi, and N. Takahashi, Phys. Rev. Lett. 55, 2676 (1985).

[3] I. Tanihata, Prog. Part. Nucl. Phys. 35, 505 (1995).

[4] I. Tanihata, Nucl. Phys. A488, 113c (1988).

[5] I. Tanihata, T. Kobayashi, O. Yamakawa, S. Shimoura, K. Ekuni, K. Sugimoto, N. Takahashi, T. Shimoda, and H. Sato, Phys. Lett. B206, 592 (1988).

[6] I. Tanihata, D. Hirata, T. Kobayashi, S. Shimoura, K. Sigimoto, and H. Toki, Phys. Lett. B289, 261 (1992).

[7] P. G. Hansen, A. S. Jensen, and B. Jonson, Annu. Rev. Nucl. Sci. 45, 591 (1995).

[8] J. Dobaczewski, I. Hamamoto, W. Nazarewicz, and J. A. Sheikh, Phys. Rev. Lett. 72, 981 (1994).

[9] R. F. Casten and B. M. Sherill, Prog. Part. Nucl. Phys. 45, S171 (2000); see also the special issue of Nucl. Phys. A693, Nos. 1-2 (2001).

[10] G. M. Ter-Akopian et al., Phys. Lett. B426, 251 (1998); G. M. Ter-Akopian et al., in Proceedings of the Symposium on Fundamental Issues in Elementary Matter, Bad Honnef, Germany, 2000, edited by W. Greiner (EP Systems Bt., Debrecen, 2001), p. 371.

[11] R. Wolski et al., JINR Preprint E15-98-284, 1998; Phys. Lett. B467, 8 (1999); S. Stepantsov et al., Phys. Lett. B542, 35 (2002).

[12] L. Giot et al., Nucl. Phys. A738, 426 (2004).

[13] K. Rusek, K. W. Kemper, and R. Wolski, Phys. Rev. C 64, 044602 (2001).

[14] V. Lapoux et al., Phys. Lett. B517, 18 (2001).

[15] M. D. Cortina-Gil et al., Nucl. Phys. A616, 215c (1997).

[16] A. Lagoyannis et al., Phys. Lett. B518, 27 (2001).

[17] M. D. Cortina-Gil et al., Phys. Lett. B371, 14 (1996).

[18] A. A. Korsheninnikov et al., Nucl. Phys. A616, 189c (1997).

[19] A. A. Korsheninnikov et al., Nucl. Phys. A617, 45 (1997).

[20] G. D. Alkhazov et al., Nucl. Phys. A712, 269 (2002).
[21] P. Egelhof, Prog. Part. Nucl. Phys. 46, 307 (2001).

[22] P. Egelhof, Eur. Phys. J. A 15, 27 (2002).

[23] S. R. Neumaier et al., Nucl. Phys. A712, 247 (2002).

[24] P. Egelhof, O. Kisselev, G. Münzenberg, S. R. Neumaier, and H. Weick, Phys. Scr. T104, 151 (2003), and references therein.

[25] L. L. Chulkov, C. A. Bertulani, and A. A. Korsheninnikov, Nucl. Phys. A587, 291 (1995)

[26] R. Crespo, J. A. Tostevin, and R. C. Johnson, Phys. Rev. C 51, 3283 (1995).

[27] M. V. Zhukov, B. V. Danilin, D. V. Fedorov, J. M. Bang, I. J. Thompson, and J. S. Vaagen, Phys. Rep. 231, 151 (1993).

[28] M. V. Zhukov, A. A. Korsheninnikov, and M. H. Smedberg, Phys. Rev. C 50, R1 (1994).

[29] M. Avrigeanu, G. S. Anagnostatos, A. N. Antonov, and J. Giapitzakis, Phys. Rev. C 62, 017001 (2000).

[30] M. Avrigeanu, G. S. Anagnastatos, A. N. Antonov, and V. Avrigeanu, Int. J. Mod. Phys. E 11, 249 (2002).

[31] P. J. Dortmans, K. Amos, J. Karataglidis, and J. Raynal, Phys. Rev. C 58, 2249 (1998).

[32] S. Karataglidis, B. A. Brown, K. Amos, and P. J. Dortmans, Phys. Rev. C 55, 2826 (1997).

[33] K. Amos, W. A. Richter, S. Karataglidis, and B. A. Brown, Phys. Rev. Lett. 96, 032503 (2006).

[34] P. K. Deb, B. C. Clark, S. Hama, K. Amos, S. Karataglidis, and E. D. Cooper, Phys. Rev. C 72, 014608 (2005).

[35] P. K. Deb, K. Amos, S. Karataglidis, M. B. Chadwick, and D. G. Madland, Phys. Rev. Lett. 86, 3248 (2001).

[36] P. K. Deb and K. Amos, Phys. Rev. C 67, 067602 (2003).

[37] K. Amos, P. J. Dortmans, H. V. von Geramb, S. Karataglidis, and J. Raynal, Adv. Nucl. Phys. 25, 275 (2000).

[38] H. F. Arellano and M. Girod, Phys. Rev. C 76, 034602 (2007).

[39] Proceedings of the International School of Nuclear Physics, Erice, Italy, 1999, edited by A. Faessler, Prog. Part. Nucl. Phys. 44 (2000); G. Shrieder et al., proposal for GSI RI Beam Factory (2001).

[40] T. Suda, in Challenges of Nuclear Structure, Proceedings of 7th International Spring Seminar on Nuclear Physics, edited by Aldo Covello (World Scientific, Singapore, 2002), p. 13; proposal for RIKEN RI Beam Factory (2001). 
[41] T. Katayama, T. Suda, and I. Tanihata, Phys. Scr. T104, 129 (2003).

[42] A. N. Antonov, M. K. Gaidarov, D. N. Kadrev, P. E. Hodgson, and E. Moya de Guerra, Int. J. Mod. Phys. E 13, 759 (2004).

[43] S. Karataglidis, P. J. Dortmans, K. Amos, and C. Bennhold, Phys. Rev. C 61, 024319 (2000).

[44] S. Karataglidis, B. A. Brown, K. Amos, and P. J. Dortmans, Phys. Rev. C 55, 2826 (1997).

[45] T. Suzuki, R. Kanungo, O. Bochkarev, L. Chulkov, D. Cortina, M. Fukuda, H. Geissel, M. Hellström, M. Ivanov, R. Janik, K. Kimura, T. Kobayashi, A. A. Korsheninnikov, G. Münzenberg, F. Nickel, A. A. Ogloblin, A. Ozawa, M. Pfützner, V. Pribora, H. Simon, B. Sitàr, P. Strmen, K. Sumiyoshi, K. Summerer, I. Tanihata, M. Winkler, and K. Yoshida, Nucl. Phys. A658, 313 (1999).

[46] A. N. Antonov, D. N. Kadrev, M. K. Gaidarov, E. Moya de Guerra, P. Sarriguren, J. M. Udias, V. K. Lukyanov, E. V. Zemlyanaya, and G. Z. Krumova, Phys. Rev. C 72, 044307 (2005).

[47] P. Sarriguren, E. Moya de Guerra, A. Escuderos, and A. C. Carrizo, Nucl. Phys. A635, 55 (1998).

[48] E. Moya de Guerra, P. Sarriguren, J. A. Caballero, M. Casas, and D. W. L. Sprung, Nucl. Phys. A529, 68 (1991).

[49] D. Vautherin, Phys. Rev. C 7, 296 (1973).

[50] D. R. Yennie, D. G. Ravenhall, and R. N. Wilson, Phys. Rev. 95, 500 (1954), and references therein.

[51] V. K. Lukyanov, E. V. Zemlyanaya, D. N. Kadrev, A. N. Antonov, K. Spasova, G. S. Anagnostatos, and J. Giapitzakis, Part. Nucl. Letters, No. 2[111], 5 (2002).

[52] M. Nishimura, E. Moya de Guerra, and D. W. L. Sprung, Nucl. Phys. A435, 523 (1985).

[53] P. Sarriguren, M. K. Gaidarov, E. Moya de Guerra, and A. N. Antonov, Phys. Rev. C 76, 044322 (2007).

[54] G. B. West, Phys. Rep. 18, 263 (1975); I. Sick, D. B. Day, and J. S. McCarthy, Phys. Rev. Lett. 45, 871 (1980).

[55] C. Ciofi degli Atti and G. B. West, Phys. Lett. B458, 447 (1999), and references therein.

[56] W. M. Alberico, A. Molinari, T. W. Donnelly, E. L. Kronenberg, and J. W. Van Orden, Phys. Rev. C 38, 1801 (1988).

[57] M. B. Barbaro, R. Cenni, A. De Pace, T. W. Donnelly, and A. Molinari, Nucl. Phys. A643, 137 (1998).

[58] T. W. Donnelly and I. Sick, Phys. Rev. Lett. 82, 3212 (1999).

[59] T. W. Donnelly and I. Sick, Phys. Rev. C 60, 065502 (1999).

[60] A. N. Antonov, M. K. Gaidarov, D. N. Kadrev, M. V. Ivanov, E. Moya de Guerra, and J. M. Udias, Phys. Rev. C 69, 044321 (2004).

[61] A. N. Antonov, M. K. Gaidarov, M. V. Ivanov, D. N. Kadrev, E. Moya de Guerra, P. Sarriguren, and J. M. Udias, Phys. Rev. C 71, 014317 (2005).

[62] A. N. Antonov, M. V. Ivanov, M. K. Gaidarov, E. Moya de Guerra, P. Sarriguren, and J. M. Udias, Phys. Rev. C 73, 047302 (2006); 73, 059901(E) (2006).

[63] A. N. Antonov, M. V. Ivanov, M. K. Gaidarov, E. Moya de Guerra, J. A. Caballero, M. B. Barbaro, J. M. Udias, and P. Sarriguren, Phys. Rev. C 74, 054603 (2006).

[64] A. N. Antonov, P. E. Hodgson, and I. Zh. Petkov, Nucleon Momentum and Density Distributions in Nuclei (Clarendon Press, Oxford, 1988); Nucleon Correlations in Nuclei (SpringerVerlag, Berlin/Heidelberg/New York, 1993).

[65] M. Jaminon, C. Mahaux, and H. Ngô, Phys. Lett. B158, 103 (1985); Nucl. Phys. A473, 509 (1987); O. Bohigas and S. Stringari, Phys. Lett. B95, 9 (1980).
[66] S. Frullani and J. Mougey, Adv. Nucl. Phys. 14, 1 (1984)

[67] L. Lapikás, in Proceedings of 4th Workshop on Perspectives in Nuclear Physics at Intermediate Energies, Trieste, Italy, 1989, edited by S. Boffi, C. Ciofi degli Atti, and M. M. Giannini (World Scientific, Singapore, 1989), p. 419; Inst. Phys. Conf. Ser. No. 105, 223 (1990), and references therein.

[68] J. B. J. M. Lanen et al., Nucl. Phys. A560, 811 (1993).

[69] J. M. Udias, P. Sarriguren, E. Moya de Guerra, and J. A. Caballero, Phys. Rev. C 53, R1488 (1996); J. M. Udias, J. A. Caballero, E. Moya de Guerra, J. R. Vignote, and A. Escuderos, ibid. 64, 024614 (2001).

[70] S. Boffi, C. Giusti, and F. D. Pacati, Nucl. Phys. A386, 599 (1982); E. Moya de Guerra et al., ibid. A477, 445 (1988).

[71] J. Mougey et al., Nucl. Phys. A262, 461 (1976).

[72] I. J. Thompson and Y. Suzuki, Nucl. Phys. A693, 424 (2001).

[73] J. A. Tostevin and J. S. Al-Khalili, AIP Conf. Proc. 455, 227 (1998); J. S. Al-Khalili and J. A. Tostevin, Phys. Rev. C 57, 1846 (1998).

[74] A. A. Korsheninnikov et al., Phys. Rev. Lett. 78, 2317 (1997).

[75] H. G. Bolen et al., Prog. Part. Nucl. Phys. 42, 17 (1999); F. Marechal et al., Phys. Rev. C 60, 034615 (1999).

[76] W. Horiuchi and Y. Suzuki, Phys. Rev. C 76, 024311 (2007).

[77] M. K. Gaidarov, A. N. Antonov, S. E. Massen, and G. S. Anagnostatos, JINR Rapid Commun. No. 6[80]-96, 23 (1996).

[78] A. N. Antonov, M. K. Gaidarov, M. V. Ivanov, D. N. Kadrev, G. Z. Krumova, P. E. Hodgson, and H. V. von Geramb, Phys. Rev. C 65, 024306 (2002).

[79] J. Carbonell and V. A. Karmanov, Nucl. Phys. A581, 625 (1995); J. Carbonell, B. Desplanques, V. A. Karmanov, and J.-F. Mathiot, Phys. Rep. 300, 215 (1998), and references therein.

[80] S. Stringari, M. Traini, and O. Bohigas, Nucl. Phys. A516, 33 (1990).

[81] E. Chabanat, P. Bonche, P. Haensel, J. Meyer, and R. Schaeffer, Nucl. Phys. A635, 231 (1998).

[82] J. G. Zabolitzky and W. Ey, Phys. Lett. B76, 527 (1978).

[83] P.-O. Löwdin, Phys. Rev. 97, 1474 (1955).

[84] M. V. Stoitsov, A. N. Antonov, and S. S. Dimitrova, Phys. Rev. C 47, R455 (1993); 48, 74 (1993).

[85] D. Faralli, C. Ciofi degli Atti, and G. B. West, in Proceedings of 2nd International Conference on Perspectives in Hadronic Physics, ICTP, Trieste, Italy, 1999, edited by S. Boffi, C. Ciofi degli Atti, and M. M. Giannini (World Scientific, Singapore, 2000), p. 75.

[86] C. Ciofi degli Atti and S. Simula, Phys. Rev. C 53, 1689 (1996).

[87] C. Ciofi degli Atti and G. B. West, nucl-th/9702009 (1997).

[88] M. F. Flynn, J. W. Clark, R. M. Panoff, O. Bohigas, and S. Stringari, Nucl. Phys. A427, 253 (1984).

[89] S. Fantoni and V. R. Pandharipande, Nucl. Phys. A427, 473 (1984).

[90] K. Amos, S. Karataglidis, and J. Dobaczewski, Phys. Rev. C 70, 024607 (2004).

[91] M. Beiner, H. Flocard, N. Van Giai, and P. Quentin, Nucl. Phys. A238, 29 (1975).

[92] N. Van Giai and H. Sagawa, Phys. Lett. B106, 379 (1981).

[93] M. Casas, J. Martorell, E. Moya de Guerra, and J. Treiner, Nucl. Phys. A473, 429 (1987); M. Casas, J. Martorell, and E. Moya de Guerra, Phys. Lett. B167, 263 (1986); J. Martorell and E. Moya de Guerra, Ann. Phys. (NY) 158, 1 (1984).

[94] J. A. Caballero and E. Moya de Guerra, Nucl. Phys. A509, 117 (1990); J. A. Caballero et al., ibid. A643, 189 (1998). 